\title{
THE LOGARITHMIC FUNCTION AND TRACE ZERO ELEMENTS IN FINITE VON NEUMANN FACTORS
}

\author{
LAJOS MOLNÁR
}

\begin{abstract}
In this short note we present a common characterization of the logarithmic function and the subspace of all trace zero elements in finite von Neumann factors.
\end{abstract}

Our aim is to prove the following statement.

Theorem 1. Let $\mathscr{A}$ be a von Neumann factor and $f:] 0, \infty[\rightarrow \mathbb{R}$ be a nonconstant continuous function. Set

$$
\mathscr{S}_{f}^{\mathscr{A}}=\overline{\operatorname{span}}\left\{f(A B A)-(2 f(A)+f(B)): A, B \in \mathscr{A}_{+}^{-1}\right\} .
$$

Then either we have $\mathscr{S}_{f}^{\mathscr{A}}=\mathscr{A}$ or we have $\mathscr{S}_{f}^{\mathscr{A}} \subsetneq \mathscr{A}$ in which case $\mathscr{A}$ is finite, $f=a \log$ holds with some constant $a \neq 0$, and $\mathscr{S}_{f}^{\mathscr{A}}$ equals the space of all trace zero elements of $\mathscr{A}$.

Here $\overline{\text { span }}$ stands for the closed linear span relative to the norm topology in $\mathscr{A}$. The above statement can be viewed as a common characterization of the logarithmic function and the space of all trace zero elements (and hence the trace itself) in factor von Neumann algebras of finite type. For the proof we need some preliminary preparations which follow.

We call a linear functional $l$ on an algebra $\mathscr{A}$ tracial if it satisfies $l(X Y)=l(Y X)$ for any $X, Y \in \mathscr{A}$. If $\mathscr{A}$ is a *-algebra, a linear functional $h: \mathscr{A} \rightarrow \mathbb{C}$ is called Hermitian if $h\left(X^{*}\right)=\overline{h(X)}$ holds for all $X \in \mathscr{A}$.

Assume now that $\mathscr{A}$ is a $C^{*}$-algebra. For a tracial bounded linear functional $l$ on $\mathscr{A}$, defining

$$
l_{1}(X)=(1 / 2)\left(l(X)+\overline{l\left(X^{*}\right)}\right), \quad l_{2}(X)=(1 / 2 i)\left(l(X)-\overline{l\left(X^{*}\right)}\right), \quad X \in \mathscr{A}
$$

we have Hermitian tracial bounded linear functionals $l_{1}, l_{2}$ such that $l=$ $l_{1}+i l_{2}$.

2010 Mathematics Subject Classification. 46L10.

Key words and phrases. Logarithmic function, trace, von Neumann factor of finite type.

The author was supported by the "Lendület" Program (LP2012-46/2012) of the Hungarian Academy of Sciences and by the Hungarian Scientific Research Fund (OTKA) Reg. No. K115383. 
It is well-known that every Hermitian bounded linear functional $h$ on the $C^{*}$-algebra $\mathscr{A}$ can be written as $h=\varphi-\psi$, where $\varphi, \psi$ are positive (bounded) linear functionals on $\mathscr{A}$ and the above decomposition, called Jordan decomposition, is uniquely determined by the condition $\|\varphi-\psi\|=\|\varphi\|+\|\psi\|$. See, for example, 3.2.5. Theorem in [3]. If $h$ is tracial, so are $\varphi$ and $\psi$. To see this, for any unitary element $U \in \mathscr{A}$ define $\varphi_{U}(X)=\varphi\left(U X U^{*}\right), X \in \mathscr{A}$ and define $\psi_{U}$ in a similar way. It is obvious that $\varphi_{U}, \psi_{U}$ are positive linear functionals, $\left\|\varphi_{U}\right\|=\|\varphi\|,\left\|\psi_{U}\right\|=\|\psi\|$, $\left\|\varphi_{U}-\psi_{U}\right\|=\|\varphi-\psi\|$, and we have

$$
\varphi(X)-\psi(X)=h(X)=h\left(U X U^{*}\right)=\varphi_{U}(X)-\psi_{U}(X), \quad X \in \mathscr{A} .
$$

By the uniqueness of the Jordan decomposition mentioned above, it follows that $\varphi_{U}=\varphi$ and $\psi_{U}=\psi$ implying that $\varphi, \psi$ are invariant under all unitary similarity transformations. But it is well-known that this implies that $\varphi, \psi$ are necessarily tracial. In fact, this follows from the argument given below in the paragraph containing the equation (3), or see 8.1.1. Proposition in [1] .

We can now prove the following statement which is certainly known, we present it for the reader's convenience. Recall that in any finite von Neumann algebra there is a unique center-valued positive linear functional which is tracial and acts as the identity on the center. This functional is called the trace, see 8.2.8. Theorem in [1].

Proposition 2. Assume that $\mathscr{A}$ is a von Neumann factor and $l$ is a nonzero tracial bounded linear functional on $\mathscr{A}$. Then $\mathscr{A}$ is of finite type and $l$ is a scalar multiple of the (unique) trace.

Proof. Clearly, we may assume that $l$ is Hermitian. Consider the Jordan decomposition $l=\varphi-\psi$ of $l$. As we have seen above, the positive linear functionals $\varphi, \psi$ are also tracial and one of them is necessarily nonzero.

Suppose $\omega$ is a nonzero positive tracial functional on $\mathscr{A}$. Then $\mathscr{A}$ cannot be infinite. Indeed in such a case we would have $I=P+Q$ with some projections $P, Q \in \mathscr{A}$ both equivalent to $I$. Since $\omega$ clearly takes equal values on equivalent projections, from $\omega(I)=\omega(P)+\omega(Q)$ we infer $\omega(I)=0$. By positivity this implies that $\omega$ vanishes on all projections which, by continuity and spectral theorem, would yield $\omega=0$, a contradiction. Therefore, the existence of a nonzero positive tracial functional $\omega$ on $\mathscr{A}$ implies that $\mathscr{A}$ is necessarily finite and by 8.2 .8 . Theorem in [1] this is a constant multiple of the trace.

After these preliminaries we can now present the proof of our result.

Proof of Theorem 1. Let $\mathscr{A}$ be a von Neumann factor and $f:] 0, \infty[\rightarrow \mathbb{R}$ be a nonconstant continuous function. Assume $\mathscr{S}_{f}^{\mathscr{A}}$ is not equal to the 
whole algebra $\mathscr{A}$. By Hahn-Banach theorem we have a nonzero bounded linear functional $l$ on $\mathscr{A}$ such that

$$
l(f(A B A)-(2 f(A)+f(B)))=0, \quad A, B \in \mathscr{A}_{+}^{-1} .
$$

Since $l$ is not zero and, by spectral theorem, the closed linear span of the set of all projections in $\mathscr{A}$ equals $\mathscr{A}$, it follows that we have a projection $P \in \mathscr{A}$ such that $l(P) \neq 0$. Denote $P^{\perp}=I-P$. Put $A=t P+P^{\perp}$ and $B=s P+$ $P^{\perp}$ and plug them into (1), where $t, s$ are arbitrary positive real numbers. It follows from (1) that

$$
l\left(\left(\left(f\left(t^{2} s\right)-(2 f(t)+f(s))\right) P-2 f(1) P^{\perp}\right)=0\right.
$$

and hence

$$
\left(\left(f\left(t^{2} s\right)-(2 f(t)+f(s))\right) l(P)=2 f(1) l\left(P^{\perp}\right)\right.
$$

holds for all $t, s>0$. This implies that

$$
f\left(t^{2} s\right)-(2 f(t)+f(s))=-2 c
$$

for all $t, s>0$ with some given real number $c$. This means that for $f^{\prime}=$ $f-c$ we have

$$
f^{\prime}\left(t^{2} s\right)-\left(2 f^{\prime}(t)+f^{\prime}(s)\right)=0, \quad t, s>0 .
$$

Substituting $t=s=1$ we obtain $f^{\prime}(1)=0$. Plugging $s=1$ we get $f^{\prime}\left(t^{2}\right)=$ $2 f^{\prime}(t)$ and finally we deduce

$$
f^{\prime}(t s)=f^{\prime}(t)+f^{\prime}(s), \quad t, s>0 .
$$

Considering the function $t \mapsto f^{\prime}(\exp (t))$ we obtain a continuous real function which is additive and hence linear implying that it is a scalar multiple of the identity. Consequently, $f^{\prime}$ is a scalar multiple of the logarithmic function and this yields that $f=a \log +b$ holds with some real scalars $a, b$.

Clearly, $a$ is nonzero and the equality (1) can be rewritten as

$$
l(\log (A B A)-(2 \log (A)+\log (B)))=d, \quad A, B \in \mathscr{A}_{+}^{-1}
$$

with $d=(2 b / a) l(I)$. Inserting $A=B=I$ it follows that $d=0$ and hence we obtain

$$
l(\log (A B A))=2 l(\log (A))+l(\log (B))), \quad A, B \in \mathscr{A}_{+}^{-1} .
$$

Now, the validity of the equation (2) implies that the linear functional $l$ is tracial. In fact, this is the content of Lemma 15 in [2]. For the sake of completeness we present the proof. First pick projections $P, Q$ in $\mathscr{A}$. Let

$$
A=I+t P, \quad B=I+t Q,
$$

where $t>-1$ is any real number. Easy computation shows that

$$
\begin{gathered}
A B A=(I+t P)(I+t Q)(I+t P) \\
=I+t(2 P+Q)+t^{2}(P+P Q+Q P)+t^{3}(P Q P) .
\end{gathered}
$$


Recall that in an arbitrary unital Banach algebra, for any element $a$ with $\|a\|<1$ we have

$$
\log (1+a)=\sum_{n=1}^{\infty} \frac{(-1)^{n+1} a^{n}}{n} .
$$

This shows that for suitable positive real number $\epsilon$, the elements $\log (A B A), \log A, \log B$ of $\mathscr{A}$ can be expressed by power series of $t(|t|<\epsilon)$ with algebra coefficients. In particular, considering the coefficients of $t^{3}$ on both sides of the equality (2) and using their uniqueness, we obtain the equation

$$
\begin{gathered}
l\left(P Q P-\frac{1}{2}((2 P+Q)(P+P Q+Q P)\right. \\
\left.+(P+P Q+Q P)(2 P+Q))+\frac{1}{3}(2 P+Q)^{3}\right) \\
=l\left(\frac{1}{3}(P+Q+P)\right) .
\end{gathered}
$$

Executing the operations and subtracting those terms which appear on both sides of this equation, we arrive at the equality

$$
l\left(\frac{1}{3}(P Q P)-\frac{1}{3}(Q P Q)\right)=0 .
$$

Therefore,

$$
l(P Q P)=l(Q P Q)
$$

holds for all projections $P, Q \in \mathscr{A}$. We claim that this implies that $l$ is tracial. To see this, select an arbitrary pair $P, Q$ of projections in $\mathscr{A}$, define $S=I-2 P$ and compute

$$
\begin{gathered}
l(Q+S Q S)=\frac{1}{2} l((I-S) Q(I-S)+(I+S) Q(I+S)) \\
=\frac{1}{2} l(4 P Q P+4(I-P) Q(I-P)) \\
=2 l(P Q P+(I-P) Q(I-P)) \\
=2 l(Q P Q+Q(I-P) Q)=2 l(Q) .
\end{gathered}
$$

Since the symmetries (i.e., self-adjoint unitaries) in $\mathscr{A}$ are exactly the elements of the form $S=I-2 P$ with some projection $P \in \mathscr{A}$, we obtain that $l(Q)=l(S Q S)$ holds for every symmetry $S$ and every projection $Q$ in $\mathscr{A}$. By the continuity of the linear functional $l$ and spectral theorem, we infer $l(X)=l(S X S)$ holds for any $X \in \mathscr{A}$ and symmetry $S \in \mathscr{A}$. This implies that

$$
l(S X)=l(S(X S) S)=l(X S)
$$

for all $X \in \mathscr{A}$ and symmetry $S \in \mathscr{A}$. Plainly, this gives us that $l(P X)=$ $l(X P)$ holds for every projection $P \in \mathscr{A}$. Finally, we conclude that $l(X Y)=$ 
$l(Y X)$ for all $X, Y \in \mathscr{A}$, i.e., $l$ is a nonzero tracial bounded linear functional. By Proposition 2 it follows that the factor $\mathscr{A}$ is of finite type and $l$ is a (nonzero) scalar multiple of the trace. In particular, $l(I) \neq 0$ and since $0=d=(2 b / a) l(I)$, it follows that $b=0$ yielding $f=a \log$.

To see that $\mathscr{S}_{f}^{\mathscr{A}}$ equals the space of all trace zero elements of $\mathscr{A}$ observe that above we have seen that any nonzero bounded linear functional $l$ on $\mathscr{A}$ with the property $\mathscr{S}_{f}^{\mathscr{A}} \subset \operatorname{ker} l$ is necessarily a scalar multiple of the same linear functional, namely, the trace. This implies that $\mathscr{S}_{f}^{\mathscr{A}}$ must equal the kernel of the trace, that is, it equals the space of all trace zero elements of $\mathscr{A}$. The proof of the theorem is complete.

We remark that one can easily find other variants of our result. Here we mention the following one. For any pair $A, B \in \mathscr{A}_{+}^{-1}$ of positive invertible elements, we denote by $A \# B$ their geometric mean, i.e., $A \# B=$ $A^{1 / 2}\left(A^{-1 / 2} B A^{-1 / 2}\right)^{1 / 2} A^{1 / 2}$.

Corollary 3. Let $\mathscr{A}$ be a von Neumann factor and $f:] 0, \infty[\rightarrow \mathbb{R}$ be a nonconstant continuous function. Set

$$
\mathscr{L}_{f}^{\mathscr{A}}=\overline{\operatorname{span}}\left\{f(A \# B)-(1 / 2)(f(A)+f(B)): A, B \in \mathscr{A}_{+}^{-1}\right\}
$$

Then either we have $\mathscr{L}_{f}^{\mathscr{A}}=\mathscr{A}$ or we have $\mathscr{L}_{f}^{\mathscr{A}} \subsetneq \mathscr{A}$ in which case $\mathscr{A}$ is finite, $f=a \log +b$ holds with some constants $a, b$ with $a \neq 0$, and $\mathscr{L}_{f}^{\mathscr{A}}$ equals the set of all trace zero elements of $\mathscr{A}$.

Proof. We only sketch the proof. First observe that replacing $f$ by the function $f-1$ we may and do assume that $f(1)=0$. If $\mathscr{L}_{f}^{\mathscr{A}} \subsetneq \mathscr{A}$, then we have a nonzero bounded linear functional $l$ on $\mathscr{A}$ such that

$$
l(f(A \# B)-(1 / 2)(f(A)+f(B)))=0, \quad A, B \in \mathscr{A}_{+}^{-1} .
$$

Similarly to the proof of Theorem 1 we obtain that

$$
f(\sqrt{t s})-(1 / 2)(f(t)+f(s))=0
$$

for any real numbers $t, s>0$ and we easily obtain that $f=a \log$ holds with some scalar $a$. Since $f$ is assumed to be nonconstant, it follows that $a \neq 0$, hence we have

$$
l(\log (A \# B)-(1 / 2)(\log (A)+\log (B)))=0, \quad A, B \in \mathscr{A}_{+}^{-1} .
$$

It is known that $A \# B$ is the unique solution $X \in \mathscr{A}_{+}^{-1}$ of the equation $X A^{-1} X=B$ which is usually called Anderson-Trapp theorem. Therefore, the above displayed equation is equivalent to

$$
l\left(2 \log (X)-\left(\log (A)+\log \left(X A^{-1} X\right)\right)\right)=0, \quad A, X \in \mathscr{A}_{+}^{-1} .
$$


and, replacing $A$ by $A^{-1}$, this is equivalent to

$$
l(\log (X A X)-(2 \log (X)+\log (A)))=0, \quad A, X \in \mathscr{A}_{+}^{-1} .
$$

By the proof of Theorem 1 we already know that this implies that the algebra $\mathscr{A}$ is finite and $l$ is a constant multiple of the trace. The proof can now be completed easily.

\section{REFERENCES}

1. R.V. Kadison and J.R. Ringrose, Fundamentals of the Theory of Operator Algebras, Vol II., Academic Press, 1986.

2. L. Molnár, General Mazur-Ulam type theorems and some applications, in Operator Semigroups Meet Complex Analysis, Harmonic Analysis and Mathematical Physics, W. Arendt, R. Chill, Y. Tomilov (Eds.), Operator Theory: Advances and Applications, Vol. 250, to appear.

3. G.K. Pedersen, $C^{*}$-algebras and Their Automorphism Groups, Academic Press, London New York San Francisco, 1979.

Department of Analysis, Bolyai Institute, University of SZeged, H-6720 SZEged, Aradi Vértanúk tere 1., Hungary and MTA-DE “LEndület” Functional Analysis Research Group, Institute of Mathematics, University of Debrecen, H-4010 DebreCEN, P.O. BoX 12, Hungary

E-mail address: molnarl@math.u-szeged.hu

URL: http: //www . math. unideb.hu/ molnarl/ 